\title{
From Neuroses to Neuropsychiatry: Replacing Adjectives With Science in the Study of Personality Disorders
}

\author{
By Jack M. Gorman, MD
}

Personality disorders are a most difficult and confusing group of psychiatric conditions. Often, psychiatrists complete a patient description by saying something akin to: "In addition to these diagnoses, the patient is also a little Axis II." Personality disorders exist on Axis II in the modern system (that is, in the editions since 1980's Diagnostic and Statistical Manual of Mental Disorders, Third Edition ${ }^{1}$ ), supposedly replacing what psychiatrists, and the general public alike, once called neuroses.

Saying that a patient is "a little Axis II" means that there are aspects of the patient's difficulties that are not easily explained by any of the Axis I diagnoses. For example, once a physician deals with the effects of depression, an anxiety disorder, or schizophrenia, there is still something that seems aberrant about the patient. The personality disorders are supposed to represent "enduring" features, meaning that they are supposed to be in place as long as the patient can remember and are resistant to change over time unless specific therapy is instituted. This, of course, is not always the case. Avoidant personality disorder, for example, often disappears when a patient is treated for social anxiety disorder with an antidepressant.

Personality disorders are often used by psychiatrists more as epithets than as diagnoses. A patient who is annoying, irritating, or whose mental health does not improve is labeled "borderline." A patient who does not pay the bill is labeled "antisocial." A patient who is rude to the receptionist is labeled "narcissistic." This is hardly the basis upon which medical diagnoses should be made.

Into this arena come the sobering scientific approaches of the collection of authors in this month's issue of CNS Spectrums, including Larry Siever, MD, of Mount Sinai School of Medicine in New York City, and his colleagues.
Their work shows that rigorous research into the personality disorders is possible and that CNS Spectrums is a solid venue for original research, with an impact factor that is steadily growing.

One striking impression I got from reading these articles is that personality disorders have much the same basis as all other psychiatric illnesses: there are genetic and environmental factors that operate to produce a set of well-defined emotional and behavioral characteristics. There are also aberrations in neurochemistry and validated treatments for personality disorders, such as dialectical behavioral treatment, which was pioneered by Marsha Linehan, $\mathrm{PhD}$, of the University of Seattle.

Personality disorders are not well studied among psychiatric investigators. Very few groups specialize in these conditions, in part because it is believed that such patients are difficult to care for and resistant to treatment efforts. Yet a tremendous amount of psychopathology, suffering, and disability stem from personality disorders. As treatments for depression, schizophrenia, and bipolar disorder improve, inpatient units, particularly those in chronic care facilities, such as state psychiatric centers, are increasingly being occupied by patients with severe forms of personality disorders.

Having a personality disorder is not simply being neurotic nor is it a matter of being a difficult patient or individual. Rather, personality disorders are emerging as an important area for neuropsychiatric research and for innovative treatment development. CNS

\section{REFERENCE}

1. Diagnostic and Statistical Manual of Mental Disorders. 3rd ed. Washington, DC: American Psychiatric Association; 1980. 